\title{
El declive del periodismo inmersivo en España a partir de 2018
}

\section{The decline of Immersive Journalism in Spain since 2018}

\author{
Antonio López Hidalgo. \\ Universidad de Sevilla. España. \\ lopezhidalgo@us.es \\ [CV] (1) 8 \\ Inés Méndez Majuelos. \\ Universidad de Sevilla. España. \\ imendez1@us.es \\ [CV] (1) 8 \\ Francisco J. Olivares-García. \\ Universidad de Sevilla. España. \\ folivares@us.es \\ [CV] (1) 용
}

\begin{abstract}
Cómo citar este artículo / Referencia normalizada
López Hidalgo, A., Méndez Majuelos, I. y Olivares-García, F. (2022). El declive del periodismo inmersivo en España a partir de 2018. Revista Latina de Comunicación Social, 80, 15-27. https://www.doi.org/10.4185/RLCS-2022-1536
\end{abstract}

\begin{abstract}
RESUMEN.
Introducción: Este trabajo presenta un análisis del periodismo inmersivo en el ámbito empresarial y profesional, tras la constatación de que la producción de vídeos informativos en formato $360^{\circ}$ ha decaído a partir del año 2018. Objetivos. Analizar las causas del cese de la producción de vídeos en formato $360^{\circ}$ en España a partir del año 2018, así como conocer la situación del periodismo inmersivo tal como la reflejan los últimos trabajos de investigación académica publicados en el ámbito científico. Metodología. Se analizan una serie de entrevistas semiestructuradas realizadas a los profesionales que realizaron las primeras experiencias inmersivas en España, así como los resultados de los últimos artículos publicados en el área de conocimiento de la comunicación. Resultados. La investigación académica en los últimos años muestra una situación optimista para las tecnologías inmersivas aplicadas a la comunicación; sin embargo, el interés de los medios de comunicación no parece acompañar a esta visión. Conclusiones. El escaso apoyo de los medios, la ausencia de tecnología para alcanzar una inmersión real de los usuarios, la escasa cultura visual de este tipo de productos en el público y, como consecuencia de ello, el bajo impacto en la audiencia son las claves de este descenso de la producción de piezas de periodismo inmersivo, frente al progresivo aumento en otros ámbitos como el videojuego o producciones audiovisuales de entretenimiento, en formato multimedia y de difusión o divulgación.
\end{abstract}

PALABRAS CLAVE: inmersivo; 360; realidad virtual; Medialab; innovación; realidad aumentada; transmedia.

\begin{abstract}
Introduction. This paper presents an analysis of immersive journalism in the business and professional sphere, following the finding that the production of news videos in $360^{\circ}$ format has declined as of 2018. Objectives. We will focus on analyzing the causes of the cessation of $360^{\circ}$ video production in Spain as of 2018, as well as knowing the situation of immersive journalism as reflected in the latest academic research papers published in the scientific scheme. Methodology. A series of semi-structured interviews conducted with professionals who carried out the first immersive experiences in Spain are analyzed, as well as the results of the latest articles published in the area of communication knowledge. Results. Academic research in recent years shows an optimistic situation for immersive technologies
\end{abstract}


applied to communication; however, the interest of the media does not seem to accompany this vision. Conclusions. The scarce support of the media, the absence of technology to achieve a real immersion of users, the scarce visual culture of this type of products in the public and, as a consequence, the low impact on the audience are the keys to this decline in the production of immersive journalism pieces, compared to the progressive increase in other areas such as video games or audiovisual productions for entertainment, in multimedia format and for broadcasting or dissemination.

KEYWORDS: Immersive; $360^{\circ}$; virtual reality; Medialab; innovation; augmented reality; transmedia.

\section{CONTENIDO}

1. Introducción. 2. Objetivos. 3. Metodología. 4. Resultados. 5. Conclusiones. 6. Bibliografía 8. Currículum Vitae

\section{CONTENT}

1. Introduction. 2. Objectives. 3. Methodology. 4. Results. 5. Conclusions. 6. Bibliography 8. Curriculum Vitae

\section{Introducción}

Acercar al lector a la noticia, a la experiencia de ser testigo del proceso informativo, es algo que el periodismo ha intentado durante muchos años. Encontramos las primeras experiencias de narrativas inmersivas en el testimonio de los muckrakers en los albores del siglo XX. Los reportajes de Martha Gellhorn sobre la Segunda Guerra Mundial, los trabajos de Walter Cronkite en la CBS durante los años 50, el movimiento del Nuevo Periodismo en los años 60, los experimentos con vídeo panorámico y audio envolvente (Domínguez Martín, 2017), o el periodismo gonzo de Hunter S. Thompson en los 70, son, sin duda, ejemplos de la evolución de este tipo de comunicación. Como consecuencia del boom en la creación de este tipo de productos en los medios, tanto extranjeros como españoles, la investigación científica y académica ha estudiado sus características y formatos, motivada por la hibridación y la multidisciplinariedad que surge de la unión de la narrativa periodística y la tecnología transmedia.

La confluencia del relato periodístico y las nuevas tecnologías han dado lugar a la generalización de términos como productos en realidad virtual o aumentada, así como los que se refieren a los usos de esas tecnologías en formato multimedia, crossmedia o transmedia. La narrativa transmedia es aquella comunicación multimedia e hipertextual basada en la transmisión de una pieza periodística a través de diferentes medios y plataformas, que incorpora la participación del receptor que desde un universo narrativo inicial accede a múltiples formas de expresión e historias paralelas que amplifican ese contenido originario, asimilando los formatos expresivos de cada uno de ellos. En este proceso es fundamental el papel de la industria tecnológica y el mercado de la cultura y la creación de contenidos (Jenkins, 2003; Scolari, 2013). De esta forma, la narrativa periodística transmedia, aprovechando los recursos interactivos que ofrecen las tecnologías de la información, busca fomentar la participación del usuario/espectador quien se convierte, de esta forma, en parte de las estrategias de difusión de la pieza, accediendo y creando narrativas que complementan el discurso periodístico básico o inicial (López Hidalgo, 2016). Supone, además, un reto para los periodistas, desplegando su trabajo en diferentes medios y plataformas, y aportando a su audiencia información y una formación básica para el mundo que les rodea (Gonçalves y Dos Santos, 2013).

El periodismo inmersivo en el entorno virtual es aquel en el que el lector/espectador/usuario recibe la información como algo real. No se trata sólo de crear una sensación de presencia o de elegir el punto de vista, sino de sentirse protagonista y vivir una experiencia de inmersión como si fuera real. En este sentido, debemos remitirnos a la conceptualización de inmersión y presencia de Slater y Wilbur (1997) y que remiten a la necesidad de aunar un alto nivel tecnológico al servicio de la creación de contenidos y su conexión con la cognición (Heeter, 2003) para conseguir las sensaciones y la identificación del espectador con el entorno y acciones propuestas (Mateer, 2017).

Comprobamos en este estudio cómo las narrativas transmedia y del periodismo inmersivo se han convertido en un objeto de investigación de gran interés en el ámbito de la comunicación. Las principales líneas de investigación que encontramos corresponden al desarrollo de un marco conceptual propio; la revisión de la evolución de la tecnología transmedia y cómo ésta hace evolucionar la narrativa perio- 
dística y, como es lógico, al análisis de los productos inmersivos que vemos en los medios de comunicación y el impacto que provoca en sus usuarios.

En el ámbito científico podemos encontrar otras definiciones y descripciones del periodismo inmersivo. La primera fue la de Nonny de la Peña, quien inicia el camino de la investigación en periodismo inmersivo, identificándolo como aquella "producción de noticias en una forma que permite a las personas obtener experiencias en primera persona sobre eventos o situaciones descritas en las historias periodísticas" (De la Peña et al., 2010). Eva Domínguez Martín (2015) plantea dos aspectos esenciales del relato inmersivo. Por un lado, retomando a De la Peña en la obra citada anteriormente, el sentimiento de ser parte de la acción, lo que denomina el "estar ahí". Y, por otro, la interacción del espectador con la noticia. La inmersión del usuario, de esta forma, se logra con técnicas que van desde el uso de una cámara de vídeo 360 grados hasta complejos escenarios de realidad virtual, en los que se puede lograr un hiperrealismo gráfico que, junto al uso de la estética del videojuego (Domínguez Martín, 2015) y la narrativa cinematográfica logran un efecto de presencia muy completo.

La primera aplicación profesional de periodismo inmersivo fue la publicación de Harvest of Change en The Moine Register, en 2014, donde ya se puede observar un uso no experimental en los medios de comunicación (Barreda Ángeles, 2018; Benítez de Gracia y Pérez Seijo, 2018). Entre 2016 y 2018 encontramos en España producciones inmersivas de El País y El Mundo (López Hidalgo y Fernández Barrero, 2016) y otros medios que utilizan realidad virtual o vídeo 360 grados, así como canales específicos en Youtube y aplicaciones móviles para dar a conocer su producción.

Sin embargo, a partir de estos años, se ha detectado una reducción drástica de la producción y el seguimiento de la innovación de las narrativas inmersivas en los medios españoles. En este trabajo tratamos de conocer los motivos que han llevado a los medios a abandonar este tipo de producciones, ya sea por falta de financiación, desinterés del grupo de comunicación o la falta de apoyo a los profesionales o periodistas que lo desarrollaban.

\section{Objetivos}

A partir de la constatación de que los medios informativos con más producción en vídeo inmersivo abandonan el formato a finales de 2018, este estudio se plantea como objetivo principal investigar las causas del cese de la producción, especialmente cuando en otros ámbitos, por ejemplo, el educativo o el divulgativo, no sólo se ha mantenido la producción, sino que además su interés por ellas se ha ampliado, aprovechando las mejoras técnicas que se han introducido en los últimos años. Un segundo objetivo es estudiar los últimos trabajos de investigación sobre esta temática, analizando la evolución que se ha producido en su enfoque y hasta qué punto se hace referencia al cese en la producción de vídeo inmersivo en los medios informativos.

\section{Metodología}

Este trabajo se plantea responder a tres cuestiones principales, que definimos a continuación como preguntas de investigación:

Pi1 ¿Cuál fue el motivo de la alta producción de piezas de periodismo inmersivo en España durante los años 2016 a $2018 ?$

Pi2 ¿Qué ocurrió para que a partir de esta fecha encontremos menos ejemplos?

Pi3 ¿Cuál es la situación desde el punto de vista profesional y el desarrollo de estas aplicaciones en el ámbito profesional y empresarial en España?

Para responder a estas cuestiones presentamos un total de cinco entrevistas a profesionales de la comunicación relacionados con el vídeo en 360 grados. En la selección no sólo se ha tenido en cuenta la experiencia profesional de los encuestados, sino que son autores de algunos de los trabajos más citados y estudiados en la literatura académica sobre el tema y ésta es la primera vez que son entrevistados para un estudio sobre formatos virtuales. Tres de los entrevistados realizaron vídeos en formato 360 grados para El País entre los años 2016 y 2018. El País fue uno de los medios más activos en la producción de piezas de vídeo 360 grados durante los años analizados. Además, se incluye una entrevista al redactor que realizó varias producciones para el diario Sur de Málaga, uno de los medios locales más activos 
en estos años. Y se concluye con la visión de una experta en producción de contenido para realidad extendida, que sigue en activo en la actualidad. Los cinco profesionales que han participado en la investigación son:

Noemí Ramírez: directora general de producto y clientes en PRISA Noticias. Tuvo un papel destacado, como productora ejecutiva en el documental Fukushima, vidas contaminadas (01-05-2016) para El País.

Saúl Ruiz: Productor audiovisual en El País. Fue el realizador de Caso Ayotzinapa, la tumba abierta (26-09-2016) y Los desplazados que ya perdonaron (01-10-2016), entre otros para El País.

Pablo León: Redactor de El País. Autor de La dehesa enferma (16-05-2018) y Silencio se rueda (2409-2018)

Jon Sedano: Redactor del diario Sur de Málaga y profesor de la Facultad de Ciencias de la Comunicación de la Universidad de Málaga. Realizó varios reportajes en vídeo 360 grados para el diario Sur entre 2016 y 2017.

Verónica Rodríguez: Comunicadora especializada en realidad virtual y creadora de contenido en realidad extendida.

La metodología planteada para la realización de las entrevistas se ha visto influida por la situación sanitaria provocada por la pandemia de covid'19, por lo que se ha optado por la realización de entrevistas mediante correo electrónico y sesiones online (Schmidt et al., 2020). En cuanto al tipo de entrevista, se optó por el modelo de entrevista semiestructurada (Folgueiras Bertomeu, 2016; Taguenca Belmonte y Vega Budar, 2012; Vargas Jiménez, 2012) basado en 10 preguntas de carácter abierto con el objeto de ampliar los resultados con las aportaciones que los sujetos quisieran aportar. Las preguntas se centraron en cuatro variables de análisis fundamentales y se dirigían al conocimiento de los siguientes aspectos: inicio de los trabajos en sus respectivos medios; tecnología empleada y equipos utilizados; impresión sobre el nivel de recepción y satisfacción de sus usuarios sobre estas producciones; y, finalmente, visión profesional acerca de la situación y futuro del periodismo inmersivo.

Por otra parte, y desde el ámbito de la investigación científica y académica, podemos comprobar que se ha seguido reflexionando sobre este tipo de narrativas y sobre el futuro de estas. Presentamos, así, una revisión bibliográfica de carácter exploratorio y sistematizada (Codina, 2020; Pérez Seijo et al., 2020) de los resultados de investigación científica publicados en los últimos años en las revistas y editoriales de referencia del ámbito de conocimiento en Comunicación. Para la selección del marco teórico no sólo se han contemplado las publicaciones de autores españoles, aunque el estudio corresponde a los medios de comunicación nacionales, sino también extranjeros que abordan las narrativas transmedia y los usos de las tecnologías transmediáticas. Los trabajos se han filtrado, en primer lugar, de acuerdo con la temática, es decir, aquellos que se ciñen de una forma más clara al objeto de la investigación; a continuación, se realizó una búsqueda de los trabajos de los tres últimos años por sus índices de calidad (JCR y Scopus) y número de citas, en los repositorios de Google Scholar y Elsevier, creando una base de datos actualizada sobre el objeto de trabajo en Mendeley Reference Manager. Para la definición de los conceptos fundamentales se ha recurrido a la bibliografía de referencia de este ámbito del Conocimiento, consolidada a lo largo de los años por sus aportaciones a la materia.

\section{Las narrativas inmersivas en el ámbito científico}

Los últimos trabajos de investigación sobre periodismo inmersivo se están centrado en el estudio de las experiencias y de las técnicas de realidad aumentada, realidad virtual o vídeo 360 grados (Jauregui Caballero y Ortega Ponce, 2020; Pérez Seijo, 2021; Salaverría, 2019), hoy denominadas realidad extendida. El objetivo de este tipo de productos, tal como se comentó anteriormente, es el de contar una historia (Benítez de Gracia y Herrera Damas, 2018b; Paíno Ambrosio y Rodríguez Fidalgo, 2019) y despertar emociones por medio de la empatía y la inclusión del usuario (Sayán Casquino, 2020; Villa Montoya, 2018). Sin duda, estos trabajos coinciden en afirmar que el papel de la tecnología es decisivo a la hora de narrar y amplificar el universo significativo de la pieza desde el plano lingüístico, espacial, sensorial o psicológico, los cuales nos ayudan a conocer el impacto que han alcanzado los productos periodísticos inmersivos, es decir la forma en que los usuarios se ven representados y se muestra la realidad a partir de su interacción (Barreda-Ángeles, 2018; Colussi y Reis, 2020; Domínguez Martín, 2017; Jauregui Caballero y Ortega Ponce, 2020; López Hidalgo y Fernández Barrero, 2016).

La evolución del estudio de la narrativa ha llevado a los investigadores a abordar también la poten- 
cialidad del relato transmedia aplicado al periodismo, atendiendo a la variedad de lenguajes que se pueden utilizar y la maleabilidad del producto al que se pueden añadir recursos transmedia, adaptando la narrativa a diferentes segmentos de edad, así como a los usos y costumbres de estos consumidores (Caerols Mateo et al., 2020; López Hidalgo y López Redondo, 2021; Marín, 2021; Palau Sampio y Sánchez García, 2020). De esta forma, y en función de la interacción, la narrativa que mayor impacto ha provocado en el público es el de piezas que abordan el reportaje de tipo social, los webdocs que hibridan el documento interactivo y los docugames, los cuales por su parte ayudan a generar en el espectador una mayor sensación de presencia, empatía o identificación, es decir, aquellas piezas que generan mayor proximidad y afinidad en los espectadores (Benítez de Gracia y Herrera Damas, 2018b; Nielsen y Sheets, 2019; Pérez Seijo, 2017; Villa Montoya, 2018; Uskali et al., 2021).

Junto a las anteriores, otra de las líneas de investigación más importantes desarrolladas por los expertos del ámbito académico ha sido el análisis de las experiencias y proyectos desarrollados por medios de comunicación. A finales de los años 60 del siglo pasado, Iván Sutherland desarrolló, en el laboratorio Lincoln del Instituto Tecnológico de Massachusetts, un prototipo de casco que dotado de dos cámaras generaba un entorno virtual y un efecto de inmersión en el usuario (Sutherland, 1968). Estas experiencias han sido continuadas por múltiples investigadores abordando las producciones multimedia que se realizaban a partir del interés existente en la generación de contenidos con tecnología inmersiva y la puesta en marcha de laboratorios de experimentación audiovisual en muchos medios (Albalad Aiguabella et al., 2018; Barreda Ángeles, 2018; López Hidalgo, 2018; Zaragoza Fuster y García Avilés, 2018). Los estudios sobre técnicas inmersivas con periodismo 360 grados son el modelo que más se ha desarrollado hasta que ha irrumpido la realidad aumentada en los medios españoles (Willens, 2017; Benítez de Gracia y Herrera Damas, 2018a; Benítez de Gracia y Pérez Seijo, 2018; Cantero de Julián et al., 2020; Paíno Ambrosio y Rodríguez Fidalgo, 2019).

La creación de departamentos y laboratorios de innovación fue la respuesta de algunos medios de comunicación a las necesidades de innovación en tecnología y contenidos, consecuencia de la crisis económica e industrial de los medios de comunicación (Méndez Majuelos, 2020; Pozo Montes y Larrondo Ureta , 2020; Benítez de Gracia y Pérez Seijo, 2018; García Avilés, 2018; Zaragoza Fuster y García Avilés, 2018). Sin duda, uno de los más importantes laboratorios de medios en España es el Laboratorio de Radio Televisión Española, RTVE Lab. En su página web se pueden encontrar interesantes producciones como, por ejemplo, las realizadas con técnicas de narrativas inmersivas para divulgación, aunque no en el sentido periodístico definido por De la Peña (2010) y a las que ya se han hecho referencia con anterioridad.

Junto a la cadena pública, otros medios de ámbito nacional y regional han destacado en la experimentación y desarrollo puntual de esas tecnologías, aplicándolas a un lenguaje periodístico y divulgativo en general, entre los que destaca El País (Carrero y García, 2021; Barreda Angeles, 2018; Benítez de Gracia y Pérez Seijo, 2018; Caerols Mateo, et al., 2020; Gutiérrez Caneda et al., 2020; López Hidalgo y Fernández Barrero, 2016; Rocha y Pase, 2018; Sidorenko Bautista et al., 2020), y las producciones del diario Sur, El Español, La Vanguardia, El Confidencial y La Sexta (Benítez de García y Herrera Damas, 2018, p. 156), utilizando la tecnología 360 grados para vídeo de contenido informativo, social y divulgativo. Benítez de Gracia y Herrera Damas $(2021,2018)$ apuntan que varios medios locales comienzan a producir vídeo 360 grados a partir de 2016. Entre ellos destaca el diario Sur de Málaga que se convierte en el medio local que más vídeo inmersivo produce entre 2016 y 2017.

Finalmente, encontramos también estudios en los que se abordan los problemas y riesgos para el periodismo a partir de un mal uso de la tecnología inmersiva aplicada a la narrativa, como sería el caso del vacío normativo y estilístico existente aún en los medios, así como limitaciones técnicas a la hora tanto de la realización como, sobre todo, de la recepción del contenido, lo cual llevaría a que se perdiera el verdadero sentido y significado de la pieza (Benítez de Gracia y Pérez Seijo, 2018; Gutiérrez Caneda et al., 2020; Paíno Ambrosio y Rodríguez Fidalgo, 2019). En cualquier caso, los resultados de investigación presentan una perspectiva optimista tanto en las posibilidades de inmersión del espectador en el contenido, como en las características de los relatos, más cercanos a la profundización, especialización, denuncia e incluso dramatización en temas de carácter social y humano (Barreda Ángeles, 2018; Carrizo, 2021; Brannon et al., 2021; Jauregui Caballero y Ortega Ponce, 2020; Paíno Ambrosio y Rodríguez Fidalgo, 2019).

En conclusión, y retornando a los orígenes de todos estos escritos, se puede afirmar que sigue vigente 
la tesis de De la Peña (2010) acerca de que será el aprovechamiento que se haga de la tecnología el que marque la diferencia entre el periodismo inmersivo y el periodismo interactivo. Esta idea se mantiene en las nuevas investigaciones y desarrollos del periodismo inmersivo, tanto en el mundo de los MediaLab, el periodismo, la docencia o la inteligencia artificial (Manfredi Sánchez et al., 2019; Tejedor Calvo et al., 2020; Greber, 2021; López García y Vizoso, 2021; Pérez Seijo y Vizoso, 2021; Pjesivac et al., 2021) y un ejemplo de ello es el desarrollo de la realidad virtual volumétrica , que mejora la experiencia de inmersión al permitir no sólo el giro en 360 grados sobre un eje, sino el desplazamiento sobre el espacio. Esta tecnología, también conocida como 6DoF (Six Degrees of Freedom), aumenta la movilidad al permitir el movimiento hacia adelante y atrás, además de en altura, frente a sistema anterior que sólo permite los movimientos de cabeza en un eje estático (Rosi, 2021). Esta técnica se está empleando de forma habitual en videojuegos, museos y entornos educativos o empresariales. Por ejemplo, el proyecto de crear una versión en vídeo volumétrico de la Biblioteca del Trinity College en Dublín (O’Dwyer, 2021), o todos lo juegos creados para la plataforma Oculus Quest o HTC Vive que ya permiten el movimiento en 6DoF. Emblematic Group, la empresa de Nonny de la Peña, está desarrollando una tecnología de vídeo volumétrico económico llamada REACH.Love que permite que cualquier empresa o medio de comunicación pueda crear productos virtuales de última generación a bajo coste (Wolozin, 2020).

\section{Evolución del vídeo inmersivo en los medios de comunicación españoles.}

Si bien los resultados de investigación publicados en los últimos años muestran una proyección optimista de este tipo de tecnologías aplicadas a la comunicación y su impacto en el periodismo, el interés de los medios parece que va por un camino diferente. Esta afirmación se sustenta en las entrevistas realizadas para este trabajo a los profesionales de los principales medios de comunicación que desarrollaron estas tecnologías aplicándolas al periodismo durante los años en los que se centra este estudio, de 2016 a 2018.

Con respecto al interés inicial de los medios en el desarrollo de narrativas inmersivas a partir de la aplicación de dispositivos de realidad virtual y aumentada, si bien es cierto que los medios se adelantaron en el uso de una tecnología todavía inmadura en aquella época, estos mismos reconocen que faltó un apoyo por parte de las marcas que estaban promoviendo la tecnología, bien por ser plataformas de contenido o bien por ser fabricantes de tecnología. En el caso de que así hubiera ocurrido, los formatos inmersivos habrían aguantado más o incluso se habrían consolidado.

Los primeros productos que encontramos de estos desarrollos, como por ejemplo la producción de vídeo 360 grados, también aparecen relacionados con la disponibilidad de tecnologías de bajo costo para la producción de estos vídeos y la existencia de empresas especializadas en este tipo de producciones, que se han contratado para la realización de algunos vídeos con estructuras más complejas. En otros casos, el comienzo de la actividad está relacionado con la apuesta personal de algún redactor interesado en el tema, como fue el caso del diario Sur, uno de los medios locales con más producción de este tipo durante estos años. Un caso muy parecido lo encontramos en algunas producciones de El País de 2018 y que parten de la iniciativa de un redactor determinado, interesado por este tipo de experiencias.

Con respecto a cómo se desarrollaba este tipo de piezas en el seno del medio y cómo afectaba a la organización del trabajo, los entrevistados coinciden al afirmar que la producción de este tipo de formatos necesita, además, un equipo de profesionales expertos y supone una producción compleja que luego no siempre obtiene el resultado de audiencia esperado. El Grupo Prisa y El País insisten en la importancia de disponer de un equipo especializado con la capacidad de contar con visores específicos y de integrar un formato con una dinámica de producción altamente tecnologizada. Estos últimos aspectos, señalan los entrevistados, han sido las causas del declive o abandono, en su caso, de este tipo de producciones. Es decir, la complejidad de la producción y la ausencia de equipos dentro de los medios que se puedan dedicar a este tipo de trabajos en exclusiva. Sin embargo, no son los únicos aspectos, ya que no podemos dejar de lado que, si estas producciones hubiesen tenido un gran impacto en la audiencia, sin duda, se mantendría el interés de los medios. Santos (2019) coincide con muchas de las conclusiones que nos dan los entrevistados, aunque es más optimista porque ve un reto en lugar de un problema cuando admite la necesidad de que los medios cuenten con un equipo técnico multidisciplinar, una tecnología potente. 
La falta de un número suficiente de usuarios con dispositivos específicos para aprovechar los formatos virtuales se plantea como uno de los principales problemas para la expansión de estos vídeos. Por ejemplo, los profesionales del Grupo Prisa consultados hacen referencia al efecto de inmersión conseguidos por estos vídeos frente al que se obtiene en un videojuego, como podría ser el del posicionamiento absoluto mediante el cual, al acercar la cabeza a un objeto, el usuario se aproxima al mismo, hecho que engaña al cerebro y logra que este se sienta dentro del producto recreado. Esto con los vídeos en 360 grados tradicionales no se consigue, por lo que, aunque algunas producciones lograban crear un mayor efecto de inmersión, al final ninguno conseguía el mismo efecto que el de un videojuego o recreación en 3D. Nuevos estudios, sin embargo, también apuntan a la posibilidad de un incremento del interés por esta tecnología añadiendo, a los anteriores, recursos como la gamificación en una intersección entre el periodismo y el videojuego, el denominado newsgames en el que existe un flujo de comunicación y argumentos complejos dentro de una dinámica periodística (García Ortega y García Avilés, 2020)

La experiencia de presencia, por tanto, a juicio de los profesionales no se ha logrado totalmente, y cuando se ha conseguido nos encontramos con que la mayoría de los usuarios no tienen un hábito de consumo de contenidos audiovisuales inmersivos. Es decir, la solución podría haberse encontrado si estas producciones se hubieran dirigido a usuarios que ya conocieran el vídeo 360 grados, como por ejemplo un público más joven y habituado al uso de estas narrativas. De esta forma, no sólo se habría aprovechado mejor este tipo de formatos, sino que además se habría conectado con una generación que está más alejada del uso de medios informativos.

En este sentido, Jon Sedano, redactor del diario Sur, reconoce que la producción de este tipo de piezas audiovisuales es compleja y que al final no se consigue un respaldo de los lectores que justifique la continuación de la producción. Esta opinión es compartida por Pablo León en El País, cuando afirma que al observarse que la expectación de los consumidores no crecía, el medio dejó de incentivar la creación de este tipo de contenido, puesto que el esfuerzo en la producción no se traducía en un impacto palpable entre sus lectores. Esta idea, de ausencia de impacto en el público, ha sido ampliamente estudiada, especialmente en lo que se refiere a las experiencias en 360 grados por Benítez de Gracia y Herrera Damas $(2021,2018)$. Del mismo modo, el trabajo de Huerta et al. (2007) llama la atención no sólo sobre el consumo, sino sobre la escasa competencia digital del público para enfrentarse y entender los productos derivados de esta tecnología. Esta idea también ha sido desarrollada por Caerols et al. (2020) cuando se refiere a la irregularidad de la producción, la ausencia de normalización de muchas propuestas y la constante experimentación con diferentes tecnologías y narrativas. Estos, a juicio de los investigadores de este ámbito de conocimiento, junto a la dificultad del acceso y la dispersión de los contenidos en las múltiples plataformas o espacios (Gutiérrez Caneda, 2020), son aspectos que dificultan la creación de hábitos de consumo de productos inmersivos.

Los entrevistados coinciden en resaltar que han podido constatar que la tecnología usada para la realización de vídeo 360 grados apenas era conocida por el público, especialmente por el usuario tipo al que se destinaron los vídeos, un lector generalista. Ante esta situación contrastada de ausencia de interés desde el punto de vista empresarial y de producción tecnológica y de contenidos, el futuro de las narrativas inmersivas en los medios de comunicación españoles parece poco halagüeño. Con respecto a nuevas experiencias, destacan el gran momento que vive la realidad extensiva, que es el término usado actualmente para referirse a todos los formatos inmersivos entre los que se encuentran, no sólo el vídeo 360 grados, sino además la realidad virtual y la aumentada. Según la opinión de Verónica Rodríguez, en estos momentos, la realidad extensiva ya se está usando en todo tipo de entornos, desde científicos, artísticos o educativos, hasta comerciales como, por ejemplo, su uso en el sector inmobiliario.

Acerca del futuro de la inmersión y del vídeo 360 grados, no parece que estos se ajusten a la forma en que los lectores quieren recibir la información. Los profesionales entrevistados admiten las grandes posibilidades del formato, pero están de acuerdo en que los usuarios no se muestran atraídos por la experiencia inmersiva que plantean estas narrativas. Ante esto, manifiestan que el vídeo 360 grados en su uso periodístico no tendrá mucho futuro, previsión que está relacionada con una forma de presentar contenido audiovisual que aporta demasiada información al espectador, información que, en realidad, el usuario ni requiere ni desea. Esto nos conecta con la reflexión realizada anteriormente con respecto a la facilidad de acceso a los productos y los contenidos inmersivos. Es posible que en un futuro, con el desarrollo del metaverso que plantea Mark Zuckerberg como futuro para las redes sociales, esta percepción cambie y se vuelvan a plantear formatos informativos basados en tecnologías inmersivas. 
A la vista de los resultados de las entrevistas realizadas, podemos concluir que los profesionales entrevistados coinciden en que fue a finales de 2018 cuando los medios de comunicación dan por terminado el periodo de experimentación con las tecnologías inmersivas y, especialmente, con el vídeo 360 grados. En este último caso, el uso de este recurso se abandonará o quedará relegado a algunos vídeos de fiestas populares sin guión ni postproducción. El único medio que sí parece que mantendrá, a juicio de los entrevistados, puesto que así lo ha hecho durante estos últimos años, cierta actividad en la producción de este tipo de formatos ha sido RTVE Lab, pero más centrado en temas divulgativos que informativos.

\section{Conclusiones}

A partir del análisis de las últimas publicaciones académicas sobre el tema y las entrevistas realizadas a los profesionales, se puede establecer que lo que motivó la alta producción de piezas inmersivas en España entre 2016 y 2018, respondiendo a la Pil, fue la novedad que ofrecía esta tecnología, para presentar la información de una forma diferente, aprovechando las narrativas transmedia, con mayor profundidad y con el objeto de conectar con los hábitos de consumo de los espectadores. Si se organizan por año de publicación los productos y piezas publicadas en medios de comunicación, encontramos que es en el año 2016 el momento en el que se concentra la mayoría de la producción de contenidos inmersivos y donde se observa mayor interés por parte de los medios en la experimentación con el formato. A partir de 2018 la producción comienza a disminuir.

En cuanto a la respuesta a la Pi2, el motivo de la falta de producción a partir de 2018 se ha constatado que estas producciones surgen en un momento de experimentación, más que como resultado de una apuesta por un formato fácilmente comercializable, lo que implica que pasado el periodo de pruebas y al no obtener los resultados de audiencia o los ingresos necesarios para continuar la producción, el formato se cancela. En cualquier caso, lo que sí parece seguro es que se adelantaron a la implantación entre el público de estas tecnologías como de consumo habitual.

Sólo una pequeña parte de la producción se dedica a productos periodísticos, ya que la mayoría de los vídeos publicados únicamente muestran las posibilidades del sistema y las ventajas de la inmersión para el usuario, pero sin llegar a generar contenido informativo o evolucionar hacia productos multimedia más complejos. Los contenidos analizados en la bibliografía consultada se mueven en la frontera del periodismo y la divulgación y aunque hay muchas referencias en los marcos teóricos sobre la influencia de Nonny de la Peña, no se encuentra ningún ejemplo que siga sus pasos. Todos los vídeos analizados se centran en el uso de la tecnología y en las posibilidades del sistema, en lugar de profundizar en las capacidades del vídeo inmersivo para acercar la noticia al usuario, como sí se puede observar en la obra de De la Peña.

En resumen, la decadencia del formato estuvo motivada por, en primer lugar, el escaso apoyo de los medios, a lo que se le une la no estandarización de los dispositivos para alcanzar una inmersión real de los usuarios. Por otra parte, desde el ámbito de la recepción, la escasa cultura visual de este tipo de productos en el público y el bajo impacto en la audiencia, han sido determinantes para el abandono paulatino de este tipo de producciones en los medios de comunicación españoles.

Finalmente, la situación actual y futurible, tal como planteamos en la Pi3, tanto desde un punto de vista profesional como empresarial, es que los medios son reticentes a gastar recursos en el uso de una tecnología sin tener la seguridad de que los consumidores lo vayan a aceptar, por lo que ya ningún medio informativo español mantiene una línea activa de producción de vídeos inmersivos. En cuanto a otras áreas temáticas, la divulgación será el espacio donde estas narrativas van a seguir teniendo continuidad, como demuestran los productos que sigue publicando el Lab de RTVE, ya sea en espacios creados ex profeso para la divulgación o en espacios informativos tradicionales para explicar a la audiencia un tema especializado o algún aspecto de la actualidad complejo y con expectación garantizada, por ejemplo: catástrofes naturales, información científica o adelantos tecnológicos.

Un ejemplo ha sido la cobertura de la información de la erupción del volcán de Cumbre Vieja en la isla de La Palma, en Canarias. La televisión pública canaria, Radio Televisión Canaria, ha dado un ejemplo a medios de comunicación con quizá mayor capacidad tecnológica de cómo abordar información especializada y compleja con realidad aumentada y, sobre todo, el uso de drones en sus espacios informativos. Hemos podido ver piezas en las que el espectador podía entrar en las entrañas del volcán y ver 
la evolución de la erupción, causas y consecuencias de lo que se vivía día a día, y todo ello respetando una información de alto nivel de especialización científica. Todo un reto, sin duda, para el periodismo tanto de medios públicos como privados. Mientras, las tecnologías inmersivas continúan avanzando desde el punto de vista tecnológico y son objeto de profusas investigaciones académicas acerca de sus características y el impacto de estas en los consumidores audiovisuales, ya sea desde el ámbito periodístico, divulgativo, lúdico o profesional.

\section{Referencias}

Albalad Aiguabella, J. M., Busto Salinas, L. \& Muñiz Zúñiga, V. (2018). Nuevos lenguajes de lo audiovisual. Editorial Gedisa.

Barreda Ángeles, M. (2018). Periodismo inmersivo en España: Análisis de la primera generación de contenidos periodísticos en realidad virtual. Estudios Sobre El Mensaje Periodístico, 24(2), 11051120. https://doi.org/f749

Benítez de Gracia, M. J. \& Herrera Damas, S. (2018a). El reportaje inmersivo en vídeo en $360^{\circ}$ en los medios periodísticos españoles. Revista de Comunicación, 17(2), 66-100. https://doi.org/f75b

Benítez de Gracia, M. J. \& Herrera Damas, S. (2018b). El reportaje inmersivo en vídeo 360o: diseño de un modelo de análisis. Profesional de la información, 27(1), 149-161. https://doi.org/f75c

Benítez de Gracia, M. J. \& Pérez Seijo, S. (2018). Análisis de las narrativas inmersivas en vídeo 360o publicadas por el LabRTVE.es (2015-2017). En M. Rodríguez Castro, S. Pérez Seijo, F. Campos Freire (Eds.), La TV Pública en Europa: Innovación, Retos y Tendencias, 146, 23-49. Cuadernos Artesanos de Comunicación. https://doi.org/f75d

Benítez de Gracia, M. J. \& Herrera Damas, S. (2021). Buenas prácticas para guiar la atención del espectador en el reportaje inmersivo con vídeo en 360'. Doxa Comunicación, 32, 263-287.

https://doi.org/g4v8

Brannon, L., Gold, L., Magee, J. \& Walton, G. (2021) The Potential of Interactivity and Gamification Within Immersive Journalism \& Interactive Documentary (I-Docs) to Explore Climate Change Literacy and Inoculate Against Misinformation. Journalism Practice. https://doi.org/g4wk

Caerols Mateo, R., Sidorenko Bautista, P. \& Garrido Pintado, P. (2020). Hacia un modelo de narrativa en periodismo inmersivo. Revista Latina de Comunicación Social, 75, 341-365. https://doi.org/f75f

Cantero de Julián, J. I., Calvo Rubio, L. M. \& Benedicto Solsona, M. A. (2020). La tenue apuesta por los vídeos en 360 o en las estrategias transmedia de las televisiones autonómicas españolas. Revista Latina de Comunicación Social, 75, 415-433. https://doi.org/f75g

Carrero, O. \& García, F. (2021). Cambios en el Periodismo durante el estado de alarma en España: El uso del video en El País. Estudios sobre el Mensaje Periodístico, 27(1), 295-306.

https://doi.org/g4v7

Carrizo, N. (2021) ¿Cómo pueden aportar las tecnologías inmersivas a la educación ambiental? Pantallas. Usos y representaciones en comunicación digital. L. Ricardo Sandoval (Coord.). Ediciones del gato gris.

Codina, L. (2020). Cómo llevar a cabo revisiones bibliográficas tradicionales o sistematizadas en trabajos de final de máster y tesis doctorales. Barcelona: Universitat Pompeu Fabra, Departamento de Comunicación, Máster Universitario en Investigación en Comunicación Social (MUCS).

https://bit.ly/3Ga8Apm

Colussi, J. \& Reis, T. A. (2020). Periodismo inmersivo. Análisis de la narrativa en aplicaciones de realidad virtual. Revista Latina de Comunicación Social, 77, 19-32. https://doi.org/f75h 
De la Peña, N. (2010). Learning by doing: Seeking best practices for immersive journalism. OJR: The Online Journalism Review. https://bit.ly/3v8kNV6

De la Peña, N., Weil, P., Llobera, J., Giannopoulos, E., Pomés, A., Spaniang, B., Friedman, D., Sánchez Vives, M. V. \& Slater, M. (2010). Immersive journalism: Immersive virtual reality for the first-person experience of news. Presence: Teleoperators and Virtual Environments, 19(4), 291-301.

https://doi.org/c9wvt9

Domínguez Martín, E. (2015). Periodismo inmersivo o cómo la realidad virtual y el videojuego influyen en la interfaz e interactividad del relato de actualidad. Profesional de la información, 24(4), 413423. https://doi.org/f75j

Domínguez Martín, E. (2017). Going Beyond the Classic News Narrative Convention: The Background to and Challenges of Immersion in Journalism. Frontiers in Digital Humanities, 4.

https://doi.org/d8kc

Dos Santos, M. C. (2019). Narrativas e desenvolvimento de conteúdo imersivo: aplicação de sistemas bi e multissensoriais de realidade virtual no jornalismo. Intercom: Revista Brasileira de Ciências da Comunicação, 42(3), 133-150. https://doi.org/hbcp

Folgueiras Bertomeu, P. (2016). La entrevista. Universitat de Barcelona.

García Avilés, J. A. (2018). Resultados de la innovación en los laboratorios de medios: El caso de El Confidencial.Lab. Profesional de la información, 27(2), 359-366. https://doi.org/f75k

García Ortega, A. \& García Avilés, J. A. 2020. When journalism and games intersect: Examining news quality, design and mechanics of political newsgames. Convergence, 26(3), 517-536.

https://doi.org/g97d

Gonçalves Morales, E; Dos Santos, M (2013) O jornalismo como narrativa transmídia: reflexoes possíveis. En Periodismo Transmedia: Miradas Múltiples. UOC.

Greber, H. (2021) Immersive Journalism - The Future of the News. IEEE Conference on Virtual Reality and $3 D$ User Interfaces Abstracts and Workshops (VRW). https://doi.org/g4wd

Gutiérrez Caneda, B., Pérez Seijo, S. \& López García, X. (2020). Las secciones y Apps de RV y vídeos 360 o a examen. Estudio de caso de siete medios con impacto en Europa. Revista Latina de Comunicación Social, 75, 149-67. https://doi.org/f75m

Heeter, C. (2003). Reflections on Real Presence by a Virtual Person. Presence: Teleoperators and Virtual Environments, 12(4), 335-345. https://doi.org/cmh9vh

Huerta, G., Torres, C. \& Angulo J. (2017). La Competencia Comunicativa en el marco de la Competencia Digital. En J. Herrero y M. Trenta (Coords.), El fin de un modelo de política. Cuadernos Artesanos de Comunicación, 128. La Laguna (Tenerife): Latina. https://doi.org/10.4185/cac129

Jauregui Caballero, A. \& Ortega Ponce, C. (2020). Narrativas transmediáticas en la apropiación social del conocimiento. Revista Latina de Comunicación Social, 77, 357-372. https://doi.org/f75n

Jenkins, H. (2003). Transmedia Storytelling. Moving characters from books to films to video games can make them stronger and more compelling. MIT Technology Review. https://bit.ly/3FuPE3M

López Hidalgo, A. (2018). El periodismo que contará el futuro. Comunicación Social Ediciones y Publicaciones.

López Hidalgo, A. \& Fernández Barrero, A. (2016). Retos para la consolidación del reportaje inmersivo. Estudio de caso de las experiencias pioneras en España de El Mundo y El País. Mediatika, 15, 125-140. https://bit.ly/3pmlRF4 
López Hidalgo, A. \& López Redondo, I. (2021). Nuevos registros narrativos en el periodismo cómic. Un estudio de caso: La grieta. Profesional de la información, 30(1), e300117. https://doi.org/gj8c7g

López García, X. \& Vizoso, A. (2021). Periodismo de alta tecnología: signo de los tiempos digitales del tercer milenio. Profesional de la información, 30(3), e300301. https://doi.org/g4wf

Manfredi Sánchez, J. L., Ufarte Ruiz, M. J. \& Herranz de la Casa, J. M. (2019). Innovación periodística y sociedad digital: Una adaptación de los estudios de Periodismo. Revista Latina de Comunicación Social, 74, 1633-1654. https://doi.org/d8j9

Marín Carrillo, A. (2021). El documental inmersivo: comprender el fenómeno en los relatos de no ficción a través de una propuesta de tipología. Profesional de la información, 30(4), e300411.

https://doi.org/g4v5

Mateer, J. (2017). Directing for cinematic virtual reality: How the traditional film director's craft applies to Immersive environments and notions of presence. Journal of Media Practice, 18(1), 14-25. https://doi.org/dqrm

Méndez Majuelos, I. (2020). Nuevas narrativas en el periodismo audiovisual. Realidad aumentada en la información audiovisual en España. En A. López Hidalgo, M. J. Ruiz Acosta (Coords). El Periodismo en Tiempos de Realidad Virtual. Comunicación Social Ediciones y Publicaciones.

Nielsen, S. L. \& Sheets, P. (2019). Virtual hype meets reality: Users' perception of immersive journalism. Journalism. https://doi.org/gf69r8

O’Dwyer, N., Zerman, E., Young, G., Smolic, A., Dunne, S. \& Shenton, H. (2021). Volumetric Video in Augmented Reality Applications for Museological Narratives: A User Study for the Long Room in the Library of Trinity College Dublin. Journal on Computing and Cultural Heritage, 4(2), 1-20.

https://doi.org/hdfh

Paíno Ambrosio, A. \& Rodríguez Fidalgo, M. I. (2019). Propuesta de géneros periodísticos inmersivos basados en la realidad virtual y el vídeo en $360^{\circ}$. Revista Latina de Comunicación Social, 74, $1132-$ 1153. https://doi.org/f75p

Palau Sampio, D. \& Sánchez García, P. (2020). Recursos digitales en la narrativa periodística de actualidad: usos y limitaciones del hipertexto, el multimedia y la interactividad. Communication and Society, 33(2), 1-16. https://doi.org/f75q

Pérez Seijo, S. (2017). Immersive journalism: From audience to first-person experience of news. In Campos Freire F., Rúas Araújo X., Martínez Fernández V., López García X. (eds). Media and Metamedia Management. Advances in Intelligent Systems and Computing, vol. 503. Springer.

https://doi.org/f748

Pérez Seijo, S., Gutiérrez Caneda, B. \& López García, X. (2020). Periodismo digital y alta tecnología: de la consolidación a los renovados desafíos. Index. Comunicación: Revista científica en el ámbito de la Comunicación Aplicada, 10(3), 129-152. https://doi.org/g3gn

Pérez Seijo, S. \& Vizoso, Á. (2021). ¿Infografías en los reportajes en vídeo 360² La integración de la visualización de la información en entornos esféricos. Estudios sobre el Mensaje Periodístico, 27(2), 607-622. https://doi.org/g4wg

Pérez Seijo, S. (2021). Uso del vídeo $360^{\circ}$ por los medios nativos digitales. Análisis exploratorio de los primeros pasos en el ecosistema periodístico español. Profesional de la información, 30(3), e300304. https://doi.org/gb8r

Pjesivac, I., Wojdynski, B. W., Binford, M. T., Kim, J., \& Herndon, K. L. (2021). Using Directional Cues in Immersive Journalism: The Impact on Information Processing, Narrative Transportation, Pre- 
sence, News Attitudes, and Credibility. Digital Journalism, 1-23. https://doi.org/g4wj

Pozo Montes, Y. \& Larrondo Ureta, A. (2020). Revisión de los factores de innovación en periodismo: análisis de laboratorios de medios. Zer, 25(48), 191-210. https://doi.org/f8s2

Rocha G. \& Pase A. (2018). Virtual reality and journalism: A historical review (1992-2018). Proceedings - 2018 20th Symposium on Virtual and Augmented Reality, SVR 2018, 122-132.

https://doi.org/hbch

Rossi, S., Viola, I., Toni, L. \& P. Cesar, P. (2021). A New Challenge: Behavioural Analysis Of 6-DOF User When Consuming Immersive Media. IEEE International Conference on Image Processing (ICIP), 3423-3427. https://doi.org/hdff

Salaverría, R. (2019). Periodismo digital: 25 años de investigación. Artículo de revisión. Profesional de la información, 28(1), 1-26. https://doi.org/f75r

Sayán Casquino, Y. (2020). Nuevos escenarios narrativos desde la inmersión de la realidad virtual (VR) como una experiencia de usuario en el relato. RISTI - Revista Ibérica de Sistemas e Tecnologías de Informacao, E35, 1-16. 1 https://bit.ly/2XyxddG

Scolari, C. A. (2013) Narrativas Transmedia: Cuando Todos Los Medios Cuentan. Deusto.

Sidorenko Bautista, P., Herranz de la Casa, J. M. \& Molina Díez, M. (2020). Evolución del periodismo inmersivo en España, desde su inicio hasta la pandemia por COVID-19. Estudos em Comunicação, 31, 69-94. https://bit.ly/3b0v8dD

Slater, M. \& Wilbur, S. (1997) A Framework for Immersive Virtual Environments (FIVE): Speculations on the Role of Presence in Virtual Environments. Presence: Teleoperators and Virtual Environments, 6(6), 603-616. https://doi.org/gd3zdp

Schmidt B., Palazzi A. \& Piccinini C. (2020) Online interviews: potential and challenges for data collection in the context of the COVID-19 pandemic. Revista Família, Ciclos de Vida e Saúde no Contexto Social, 8(4), 960-966. https://doi.org/hbb4

Sutherland, I. E. (1968). A Head-mounted Three Dimensional Display. Proceedings of the December 9-11, 1968, Fall Joint Computer Conference, Part I, 57-764. https://bit.ly/3E1cWgz

Taguenca Belmonte, J. A. \& Vega Budar, M. R. (2012). Técnicas de investigación social. La entrevista abierta y semidirectiva. Revista de investigación en ciencias sociales y humanidades, Nueva Época, 1(1). https://bit.ly/3m3Ckfx

Tejedor Calvo, S., Romero Rodríguez, L. M., Moncada Moncada, A. J. \& Alencar Dornelles, M. (2020). Periodismo que cuenta el futuro: posibilidades y escenarios periodísticos para la realidad aumentada. Profesional de la información, 29(6), 1-13. https://doi.org/f75v

Uskali, T., Gynnild, A., Jones, S., \& Sirkkunen, E. (2021). Immersive Journalism as Storytelling: Ethics, Production, and Design. Taylor \& Francis.

Vargas Jiménez, I. (2012) La entrevista en la investigación cualitativa: nuevas tendencias y retos. Revista Electrónica Calidad en la Educación Superior, 3(1), 119-139. https://doi.org/hbc3

Villa Montoya, M. I. (2018). Narrativas inmersivas para comunicadores. Realidad virtual, aumentada y mixta en propuestas audiovisuales de ficción y no ficción. Comunicación, 39, 7-12. https://doi.org/ $\mathrm{f} 75 \mathrm{w}$

Willens, M. (2017). One year in: What The New York Times learned from its 360-degree video project, The Daily 360. Digiday, 14 noviembre. https://bit.ly/3m20WVH 
Wolozin, S. (2020). The World Is 3D and Media Should Be Too. Nonny de la Peña reflects on her experience as a VR pioneer. Immerse News, 29 julio. https://bit.ly/3etwgaW

Zaragoza Fuster, T. \& García Avilés, J. A. (2018). Desarrollo de la innovación periodística en la televisión pública: El caso del RTVE Lab. Hipertext.Net: Revista Académica Sobre Documentación Digital y Comunicación Interactiva, 0(17). https://doi.org/f75x.

\section{AUTORES:}

\section{Antonio López Hidalgo \\ Universidad de Sevilla}

Catedrático de Periodismo en la Facultad de Comunicación de la Universidad de Sevilla y director del departamento de Periodismo II. Director del grupo de investigación Narrativas Periodísticas y Tecnologías Emergentes. Cuenta con una amplia producción científica en el ámbito de las Nuevas Tecnologías de la Comunicación y la Información, medios de comunicación social y géneros periodísticos, así como en narrativas emergentes y transmedia.

lopezhidalgo@us.es

Indice H: 15

Orcid ID: https://orcid.org/0000-0003-1595-7043

Google Scholar: https://scholar.google.es/citations?user=7MxHFrkAAAAJ\&

\section{Inés Méndez Majuelos}

Universidad de Sevilla

Profesora Contratada Doctora en la Facultad de Comunicación de la Universidad de Sevilla. Pertenece al grupo de investigación en Narrativas Periodísticas y Tecnologías Emergentes. Sus líneas de investigación son la Historia y la Innovación en narrativas periodísticas, así como el análisis de impacto de la investigación en comunicación social en la sociedad. Cuenta con experiencia en la gestión de proyectos de investigación tanto del Plan Nacional y los Programas de Investigación de la Unión Europea, como el $7^{\circ}$ Programa Marco y Horizonte 2020.

imendez1@us.es

Índice H: 2

Orcid ID: https://orcid.org/0000-0003-3237-3867

Google Scholar: https://scholar.google.com/citations?user=n_YRxooAAAAJ

\section{Francisco J. Olivares-García}

Universidad de Sevilla

Profesor Asociado en la Facultad de Comunicación de la Universidad de Sevilla. Doctor en Periodismo, durante diez años fue director de Máster en Comunicación Digital del Instituto Universitario de Postgrado, formado por las universidades Carlos III, Autónoma de Barcelona y Alicante, junto a Santillana Formación. Realizador de Canal Sur Radio y Televisión desde 1989. Experto en Gestión Multimedia y Narrativas digitales en la Dirección Técnica de Radio y Televisión de Andalucía. Pertenece al grupo de investigación en Narrativas Periodísticas y Tecnologías Emergentes.

folivares@us.es

Indice H: 3

Orcid ID: https://orcid.org/0000-0003-1475-130X

Google Scholar: https://scholar.google.es/citations?user=aS4A97YAAAAJ\&hl 